\title{
Spectrum Pricing Games with Random Valuations of Secondary Users
}

\author{
Gaurav S. Kasbekar and Saswati Sarkar
}

\begin{abstract}
We analyze price competition among primary users in a Cognitive Radio Network (CRN), in which there are a random and unknown number of secondary users. In every slot, each primary has unused bandwidth with some probability, which it would like to lease to a secondary user, and must set a price for this bandwidth. The valuations of the secondary users for unit bandwidth are independent and identically distributed random variables. We analyze this price competition as a game and explicitly compute a Nash Equilibrium (NE), which we show to be unique in the class of symmetric NE. We show that randomness in the valuations of the secondary users results in significant structural differences in the strategies of the primaries in the $\mathrm{NE}$ compared to the case in which the valuations of the secondaries are constants.
\end{abstract}

\section{INTRODUCTION}

The emerging cognitive radio technology [1] promises efficient usage of the available radio spectrum. In cognitive radio networks (CRNs), there are two types of spectrum users: (i) primary users who lease portions (channels or bands) of the spectrum directly from the regulator, and (ii) secondary users who lease channels from primaries and can use a channel when it is not in use by the primary. Time is slotted, and in every slot, each primary has unused bandwidth with some probability, which it would like to sell to secondaries. Now, secondaries buy bandwidth from the primaries that offer it at a low price, which results in price competition among the primaries. If a primary quotes a low price, it will attract buyers, but will earn lower profit per sale. This is a common feature of an oligopoly [19], in which multiple firms sell a common good to a pool of buyers. Price competition in an oligopoly is naturally modeled using game theory [18], and has been extensively studied in economics using, for example, the classic Bertrand game [19] and its variants.

However, a CRN has several distinguishing features, which makes the price competition very different from oligopolies encountered in economics. For example, in every slot, each primary may or may not have unused bandwidth available. Also, the number of secondaries will be random and not known apriori as each secondary may be a local spectrum provider or even a user shopping for spectrum in a futuristic scenario, e.g., users at airports, hotspots, etc. Thus, each primary who has unused bandwidth is uncertain about the number of primaries from whom it will face competition as well as the demand for bandwidth; it may only have access to imperfect information such as statistical distributions about either. A low price will

G. Kasbekar and S. Sarkar are with the Department of Electrical and Systems Engineering at University of Pennsylvania, Philadelphia, PA, U.S.A. Their email addresses are kgaurav@seas.upenn.edu and swati@seas.upenn.edu respectively. result in unnecessarily low revenues in the event that very few other primaries have unused bandwidth or several secondaries are shopping for bandwidth, because even with a higher price the primary's bandwidth would have been bought, and vice versa.

Also, each secondary has a certain valuation for bandwidth, and it leases out bandwidth from a primary only if the price is less than or equal to its valuation. Further, the valuations of different secondaries may possibly be different and they may be unknown to the primaries. Since whether or not a primary's bandwidth is sold depends on the valuations of the secondaries, primaries must take into account the distributions of the secondaries' valuations while choosing their prices.

Pricing related issues have been extensively studied in the context of wired networks and the Internet; see [10] for an overview. Price competition among spectrum providers in wireless networks has been studied in [11], [12], [13], [15], [16], [17]. Specifically, Niyato et. al. analyze price competition among multiple primaries in CRNs [16], [17]. However, neither uncertain bandwidth availability nor the issue of random and unknown valuations of secondaries is modeled in any of the above papers. Also, most of these papers do not explicitly find a Nash Equilibrium (NE) (exceptions are [12], [16]). Our model incorporates uncertain bandwidth availability and the issues of a random number of secondaries and random and unknown valuations of secondaries, which makes the problem challenging; despite this, we are able to explicitly compute a NE. In the economics literature, the Bertrand game [19] and several of its variants [5], [6], [8], [9] have been used to study price competition. For example, Osborne et al [5] consider price competition in a duopoly, when the capacity of each firm is constrained.

The closest to our work are [8], [9], which analyze price competition where each seller may be inactive with some probability, as also our prior work [21], [22], [23] in which we analyzed price competition in a CRN. However, the results in [8], [9] are restricted to the case of one buyer, those in [22] to the case of a constant and known number of secondaries and in [22], [23] and for the most part in [21], it is assumed that the valuations of all the secondaries are constant and equal and known to the primaries. In [8], [9], [21], the case in which the valuation is a random variable is considered only for the case of a single buyer and under some restrictive assumptions on the distribution function of its valuation. In particular in [21], it is assumed that the distribution function $G($.$) is continuous, the$ function $g(x)=(x-c)(1-G(x))$ has a unique maximizer $v_{T}>c$ and that the function $g($.$) is strictly increasing on$ the interval $\left[c, v_{T}\right]$, where $c$ is the minimum price set by any 
seller. In [8], [9], in addition to the above assumptions, it is also assumed that the function $g($.$) is differentiable on \left[c, v_{T}\right]$.

In this paper, we consider a CRN with multiple primaries and a random and unknown number of secondaries, in which each primary has unused bandwidth in a slot with some probability. The valuations of the secondaries are random and possibly unequal and independently drawn from a common distribution. Also, we relax the restrictive assumptions on the common distribution function of the valuations of the secondaries made in [8], [9], [21], and only assume that the distribution function is continuous and that the valuations lie in some range $[\underline{v}, \bar{v}]$ with probability 1 , where $c<\underline{v}<\bar{v}$. Note that these assumptions are satisfied by most distribution functions of valuations in practice. The presence of a random and unknown number of secondaries and the relaxation of the assumptions in [8], [9], [21] on the distribution function of valuations makes the analysis much more challenging. Also, since prices can take real values, the strategy sets of players are continuous. In addition, the utilities of the primaries are not continuous functions of their actions. Thus, classical results, including those for concave and potential games, do not establish the existence and uniqueness of a Nash Equlibrium (NE) in the resulting game, and there is no standard algorithm for finding a NE. In spite of this, we explicitly compute a $\mathrm{NE}$ and show that it is unique in the class of symmetric NE. Our analysis yields several insights, e.g., randomness in the valuations of the secondaries results in significant structural differences in the strategies of the primaries in the NE compared to the case in which the valuations of the secondaries are constants.

In Section II, we describe the model and problem definition, and briefly overview the analysis of the constant valuation case in [21]. Next, for simplicity, we first explicitly compute a symmetric $\mathrm{NE}$ and prove its uniqueness in the case where there is only one secondary in Section III and later generalize our results to a random and unknown number of secondaries in Section IV. The structure of the symmetric NE provides several insights, which we discuss in Section V. We provide numerical studies in Section VI and conclude in Section VII.

\section{Model, Problem Definition AND BACKGRound}

\section{A. Model}

Suppose there are $n \geq 2$ primaries and $K$ secondaries in a region. Each primary owns 1 channel in the region, which corresponds to 1 unit of bandwidth, and each secondary is a customer who requires 1 unit of bandwidth. The number of secondaries, $K$, is a random variable that takes values in the range $\left\{0, \ldots, k_{\max }\right\}$ for some finite constant $k_{\max }$ and has the probability mass function (p.m.f.) $P(K=k)=\alpha_{k}$. The primaries know the p.m.f. of $K$, but not its value. To avoid trivialities, we assume that $P(K>0)>0$, i.e. $\alpha_{0}<1$.

Time is divided into slots of equal duration. In every slot, each primary does not use its channel, i.e. has unused bandwidth, with probability (w.p.) $q \in(0,1)$. For tractability, we assume that this probability is the same for all the primaries. A primary $i$ who has unused bandwidth in a slot can lease it out to a secondary for the duration of the slot, in return for an access fee of $p_{i}$. Leasing in a slot incurs a cost of $c \geq 0$. This cost may arise, for example, if the secondary uses the primary's infrastructure to access the Internet.

Let $v_{j}, j \in\{1, \ldots, K\}$, denote the valuation of secondary $j$ for 1 unit of bandwidth- secondary $j$ is willing to buy bandwidth only at a price that is less than or equal to $v_{j}$. The valuations $v_{1}, \ldots, v_{K}$ of the secondaries for 1 unit of bandwidth are independent and identically distributed (i.i.d.) random variables with distribution function (d.f.) ${ }^{1} G(x)=$ $P\left(v_{j} \leq x\right)$. We assume that $G($.$) is continuous and G(\underline{v})=0$, $G(\bar{v})=1$, where $c<\underline{v}<\bar{v}$. Thus, the valuation of each secondary lies in the range $[\underline{v}, \bar{v}]$ w.p. 1 . Note that in practice, the valuations of secondaries are upper bounded, and hence there always exists some finite upper bound $\bar{v}$. The assumption $\underline{v}>c$ means that a secondary's valuation is always greater than the cost that the primary incurs; so there always exists a price at which trade is profitable to both the primary and the secondary.

In general, secondaries buy bandwidth from the primaries who charge the lowest prices; we will specify the exact rule that we consider for matching primaries with secondaries later in Sections III and IV-A.

\section{B. Game Formulation}

We formulate the above price competition among primaries as a game, which is any situation in which multiple individuals called players interact with each other, such that each player's welfare depends on its own action as well as the actions of others [19]. In our model, the primaries are the players, and the action of primary $i$ is the price $p_{i}$ that it chooses ${ }^{2}$.

In general, the utility or payoff of a player in a game is a numerical measure of its satisfaction level [19]. In our game, the utility of primary $i$ is defined to be 0 if it has no unused bandwidth. Let $u_{i}\left(p_{1}, \ldots, p_{n}\right)$ denote its utility if it has unused bandwidth ${ }^{3}$ and primary $j$ sets a price of $p_{j}, j=1, \ldots, n$. It is defined to be primary $i$ 's net revenue. Thus:

$u_{i}\left(p_{1}, \ldots, p_{n}\right)= \begin{cases}p_{i}-c & \text { if primary } i \text { sells its bandwidth } \\ 0 & \text { otherwise }\end{cases}$

Now, a strategy [19] for primary $i$ is a plan for choosing its price $p_{i}$. We allow each primary $i$ to choose its price randomly from a set of prices using an arbitrary d.f. $\psi_{i}($.$) , which is referred to as the strategy of pri-$ mary $i$. The vector $\left(\psi_{1}(),. \ldots, \psi_{n}().\right)$ of strategies of the primaries is called a strategy profile [19]. Let $\psi_{-i}=$ $\left(\psi_{1}(),. \ldots, \psi_{i-1}(),. \psi_{i+1}(),. \ldots, \psi_{n}().\right)$ denote the vector of strategies of primaries other than $i$. Let $E\left\{u_{i}\left(\psi_{i}(),. \psi_{-i}\right)\right\}$ denote the expected utility of primary $i$ when it adopts strategy $\psi_{i}($.$) and the other primaries adopt \psi_{-i}$.

\footnotetext{
${ }^{1}$ Recall that the distribution function (d.f.) [20] of a random variable (r.v.) $X$ is the function:

$$
F(x)=P(X \leq x), \quad x \in R
$$

where $R$ is the set of real numbers.

${ }^{2}$ If primary $i$ has no unused bandwidth, it does not matter what price $p_{i}$ it sets. Yet, for convenience, we speak of $p_{i}$ as being its action.

${ }^{3}$ If instead, $u_{i}\left(p_{1}, \ldots, p_{n}\right)$ were defined to be primary $i$ 's net revenue, unconditional on whether it has unused bandwidth or not, then its expected utility in the game analysis would be scaled everywhere by $q$.
} 
We use the Nash Equilibrium (NE) solution concept, which has been extensively used in game theory in general and wireless network applications in particular as a prediction of the outcome of a game. Several arguments have been proposed in the literature for using $\mathrm{NE}$ as a solution concept, e.g. it is a necessary condition if there is a unique predicted outcome to a game, a strategy profile can be a "focal point" only if it is a NE etc. (see Section 8.D in [19] for a detailed discussion). A NE is a strategy profile such that no player can improve its expected utility by unilaterally deviating from its strategy [19]. Thus, $\left(\psi_{1}^{*}(),. \ldots, \psi_{n}^{*}().\right)$ is a NE if for each primary $i$ :

$$
E\left\{u_{i}\left(\psi_{i}^{*}(.), \psi_{-i}^{*}\right)\right\} \geq E\left\{u_{i}\left(\widetilde{\psi}_{i}(.), \psi_{-i}^{*}\right)\right\}, \forall \widetilde{\psi}_{i}(.)
$$

When players other than $i$ play $\psi_{-i}, \psi_{i}^{*}($.$) maximizes i$ 's expected utility and is thus its best-response [19] to $\psi_{-i}$.

\section{Symmetric NE}

Since the bandwidth availability probability of each primary is the same (equal to q), the game in Section II-A is a symmetric game, which is one in which all players have the same parameters, action sets and utility functions.

We focus on a specific class of NE, known as symmetric $N E$. A NE $\left(\psi_{1}^{*}(),. \ldots, \psi_{n}^{*}().\right)$ is a symmetric NE if all players play identical strategies under it, i.e., $\psi_{1}^{*}()=.\psi_{2}^{*}()=.\ldots=\psi_{n}^{*}($.$) .$ In practice it is challenging to implement any other NE- the simple example of two primaries and a NE of $\left(\psi_{1}^{*}(),. \psi_{2}^{*}().\right)$ elucidates the inherent complications in the current context. If $\psi_{1}^{*}(.) \neq \psi_{2}^{*}($.$) , then since players have the same action sets,$ utility functions and probability of having unused bandwidth (i.e., the game is a symmetric game), $\left(\psi_{2}^{*}(),. \psi_{1}^{*}().\right)$ also constitutes a NE. If player 1 knows that player 2 is playing $\psi_{2}^{*}().\left(\psi_{1}^{*}(\right.$.$) respectively), it would choose the best response$ $\psi_{1}^{*}().\left(\psi_{2}^{*}(\right.$.$) respectively), but it cannot know player 2's choice$ between the two options without explicitly coordinating with it, which is again ruled out due to the competition between the two. Under symmetric NE, all players play the same strategy, and thus this quandary is somewhat limited- symmetric NE has indeed been advocated for symmetric games by several game theorists [4]. The natural question now is whether there exists at least one symmetric NE, and also whether there is a unique symmetric NE (only uniqueness will eliminate the above quandary). Note that some symmetric games are known to have multiple symmetric NE. For example, consider the simple "Meeting in New York game" [19] with two players, where each player can either be at Grand Central or at Empire State Building, and both receive unit utility if they meet and zero utility otherwise. The strategies where each player is at Grand Central, and where each player is at Empire State Building, both constitute symmetric NE. Our goal is to explicitly compute a symmetric $\mathrm{NE}$ and to show its uniqueness in our context.

\section{Symmetric NE in the Constant Valuation Case}

In our prior work [21], we analyzed price competition among multiple primaries when the valuation of each secondary is the same and equals a constant $v>c$ that is known to the primaries (the model in [21] is otherwise as in the present paper). In this subsection, we briefly summarize the structure of the symmetric NE that we computed for the constant $v$ model in [21]; later, in Section V, we will contrast it with the symmetric NE that we will find for the model in the present paper.

Let $4 w(q, n)=\sum_{k=0}^{k_{\max }} \alpha_{k} \sum_{i=k}^{n-1}\left(\begin{array}{c}n-1 \\ i\end{array}\right) q^{i}(1-$ $q)^{n-1-i}$. It is easy to check that $w(q, n)$ is the probability that $K$ or more out of $n-1$ primaries have unused bandwidth. Also, let $\tilde{p}=v-w(q, n)(v-c)$. In [21], we showed that in the constant valuation model, there is a unique symmetric NE; in this NE, each primary selects prices only in the range $[\tilde{p}, v]$. Also, it randomizes over the prices in $[\tilde{p}, v]$ using a continuous d.f. $\psi($.$) , which is strictly increasing on [\tilde{p}, v]$ and has been explicitly computed (see Theorem 2 and Remark 1 in [21]). Note that the symmetric NE price selection strategy $\psi($.$) is$ contiguous in the sense that it selects prices only in the interval $[\tilde{p}, v]$, and every sub-interval of this interval is selected with positive probability.

\section{E. Pseudo-price}

For convenience, we introduce the notion of a "pseudoprice". The pseudo-price of primary $i \in\{1, \ldots, n\}$, denoted as $p_{i}^{\prime}$, is the price it selects if it has unused bandwidth and $p_{i}^{\prime}=\bar{v}+1$ otherwise ${ }^{5}$. Let $\psi_{i}($.$) (respectively, \phi_{i}($.$) ) be the$ d.f. of $p_{i}$ (respectively, $p_{i}^{\prime}$ ). For $c \leq x \leq \bar{v}, p_{i}^{\prime} \leq x$ for a primary $i$ iff it has unused bandwidth and sets a price $p_{i} \leq x$. So $\phi_{i}(x)=q P\left(p_{i} \leq x\right)=q \psi_{i}(x)$. Thus, $\psi_{i}($.$) and \phi_{i}($. differ only by a constant factor on $[c, \bar{v}]$ and we use them interchangeably wherever applicable.

\section{ONE SECONDARY}

In this section, for simplicity, we find a symmetric NE and prove its uniqueness for the case in which there is only one secondary, i.e. $K=1$ w.p. 1 . This secondary buys bandwidth from the primary who quotes the lowest price, provided this price is less than or equal to its valuation. In Section IV, we generalize our results to allow for multiple and a random number of secondaries.

In Section III-A, we will explicitly compute a symmetric $\mathrm{NE}$ and in Section III-B show that it is the unique symmetric NE.

\section{A. Explicit Computation of Symmetric NE}

Consider a symmetric NE under which every primary uses the price selection strategy $\psi($.$) , and let \phi($.$) be the corre-$ sponding pseudo-price selection strategy. In this subsection, we describe the structure of $\psi($.$) (and \phi($.$) ), and in Theorem 1$ show that it indeed constitutes a symmetric NE strategy. First, the following lemma provides a necessary condition that $\psi($. must satisfy.

Lemma 1: $\psi($.$) is continuous.$

${ }^{4}$ Recall that for integers $a, b \geq 0,\left(\begin{array}{c}a \\ b\end{array}\right)$ equals 0 if $b>a$.

${ }^{5}$ The choice $\bar{v}+1$ is arbitrary. Any other value greater than $\bar{v}$ also works. 
Proof: Suppose, to reach a contradiction, that $\psi($.$) has$ a jump ${ }^{6}$ at $x_{0}$. Fix an $i \in\{1, \ldots, n\}$. Since the secondary selects the primary who quotes the lowest price, and every primary in $\{1, \ldots, n\} \backslash i$ has a jump at $x_{0}$, for primary $i$, a price just below $x_{0}$ fetches a higher expected payoff than $x_{0}$. So $x_{0}$ is not a best response for primary $i$, which contradicts the fact that primary $i$ uses $\psi($.$) and hence has a jump at x_{0}$. The result follows.

Recall that a pure-strategy NE [18] is one in which every player (primary) selects a single action (price) w.p. 1. Lemma 1 shows that there does not exist a pure strategy symmetric NE in the present context.

Let $f_{x}(y)$ be primary $m$ 's expected payoff if it sets the price $p_{m}=x$ and

$$
P\left(p_{j}^{\prime} \leq x\right)=y, \forall j \in\{1, \ldots, n\} \backslash m .
$$

Let

$$
h(x)=f_{x}(q)
$$

and

$$
g(x)=f_{x}(0) .
$$

The following lemma provides an expression for $f_{x}(y)$ :

Lemma 2:

$$
f_{x}(y)=(x-c)(1-G(x))(1-y)^{n-1}
$$

Proof: Suppose (2) holds. If primary $m$ sets a price of $x$, it gets a payoff of $(x-c)$ if its bandwidth is sold and 0 otherwise. Also, its bandwidth is sold iff ${ }^{7}$ (i) the valuation of the secondary is $x$ or more, which happens w.p. $1-G(x)$, and (ii) no primary $j \in\{1, \ldots, n\} \backslash m$ who has unused bandwidth sets a price lower than $y$, which happens w.p. $(1-y)^{n-1}$ by (2). The result follows.

We now state some properties of $f_{x}(y)$, which are proved in the Appendix:

Lemma 3: 1) $f_{x}(y)$ is continuous in $x$ and $y$.

2) For $x \leq \underline{v}, f_{x}(y)$ is a strictly increasing function of $x$ for every fixed $y$. Also, $f_{c}(y)=0$ for every fixed $y$.

3) $h(\underline{v})=f_{\underline{v}}(q)>0$. Also, $h(x)=0$ for all $x \geq \bar{v}$.

By (3) and part 1 of Lemma 3, $h($.$) is a continuous function$ and hence has a maximizer on the compact set $[c, \bar{v}]$. Let $h_{\text {max }}=\max _{v \in[c, \bar{v}]} h(v)$ be the maximum value of $h($.$) and$

$$
v_{T}=\inf \left\{v \in[c, \bar{v}]: h(v)=h_{\max }\right\}
$$

be the infimum of the set of maximizers of $h($.$) . Since h($.$) is$ continuous, by (6), $v_{T}$ is itself a maximizer of $h($.$) on [c, \bar{v}]$. So $h\left(v_{T}\right)=h_{\max }$. By part 2 of Lemma 3 and (3), $h($.$) is$ strictly increasing on $[c, \underline{v}]$. Also, $h(\underline{v})>0$ and $h(\bar{v})=0$ by part 3 of Lemma 3. Since $v_{T}$ is the smallest maximizer of $h($.$) on [c, \bar{v}]$ :

$$
\underline{v} \leq v_{T}<\bar{v} .
$$

We will later show that the upper endpoint of the support set ${ }^{8}$ of $\psi($.$) is v_{T}$.

\footnotetext{
${ }^{6}$ A d.f. $f(x)$ is said to have a jump (discontinuity) of size $b>0$ at $x=a$ if $f(a)-f(a-)=b$, where $f(a-)=\lim _{x \uparrow a} f(x)[20]$.

${ }^{7}$ Note that by Lemma 1 , the probability that a primary sets a price of exactly $y$ is 0 .

${ }^{8}$ The support set of a d.f. is the smallest closed set such that its complement has probability zero under the d.f. [20].
}

We now state another property of the function $f_{x}(y)$, which is proved in the Appendix.

Lemma 4: For every fixed $x \in\left[c, v_{T}\right], f_{x}(y)$ is a strictly decreasing function of $y$.

Lemma 5: There exists at least one $x \in\left(c, v_{T}\right)$ such that $g(x)=h\left(v_{T}\right)$. The minimum such $x$ exists; let it be denoted by $\tilde{p}$. Then $g(x)<g(\tilde{p})=h\left(v_{T}\right) \forall c \leq x<\tilde{p}$.

Proof: Since $v_{T}$ is a maximizer of $h($.$) and by part 3$ of Lemma 3, $h\left(v_{T}\right) \geq h(\underline{v})>0$. Also, by (4) and part 2 of Lemma 3:

$$
g(c)=0<h\left(v_{T}\right) .
$$

By (3), (4) and Lemma 4:

$$
h\left(v_{T}\right)=f_{v_{T}}(q)<f_{v_{T}}(0)=g\left(v_{T}\right)
$$

By (8) and (9), $g(c)<h\left(v_{T}\right)$ and $g\left(v_{T}\right)>h\left(v_{T}\right)$. Also, $g($.) is continuous by (4) and part 1 of Lemma 3. So by the intermediate value theorem [3], there exists a solution of the equation $g(x)=h\left(v_{T}\right)$ in $\left(c, v_{T}\right)$. The minimum such solution, say $\tilde{p}$, exists because $g($.$) is continuous and hence$ the set $\left\{x: g(x)=h\left(v_{T}\right)\right\}$ is closed.

Now, suppose, to reach a contradiction, that $g\left(x^{\prime}\right) \geq h\left(v_{T}\right)$ for some $x^{\prime} \in[c, \tilde{p})$. Then by (8) and the intermediate value theorem, there exists $x^{\prime \prime}$ such that $c \leq x^{\prime \prime} \leq x^{\prime}<\tilde{p}$ and $g\left(x^{\prime \prime}\right)=h\left(v_{T}\right)$. This contradicts the fact that $\tilde{p}$ is the smallest solution of $g(x)=h\left(v_{T}\right)$. Thus, $g(x)<h\left(v_{T}\right)$ for all $x<\tilde{p}$.

By definition of $f_{x}(y)$ and by (4), if no primary in $\{1, \ldots, n\} \backslash i$ plays a price below $x$, then primary $i$ gets a payoff of $g($.$) at price x$. We will later show that primaries do not play prices below $\tilde{p}$ under the strategy $\psi($.$) , and \tilde{p}$ is a best response for every primary in the corresponding NE. So the payoff that every primary gets at a best response in the $N E$ equals $g(\tilde{p})$.

Let:

$$
C=\left\{x \in\left[\tilde{p}, v_{T}\right]: g(x) \geq g(\tilde{p})\right\} .
$$

Note that for a price in $\left[\tilde{p}, v_{T}\right] \backslash C$, primary $i$ 's payoff is less than the NE payoff and hence each primary plays prices in $\left[\tilde{p}, v_{T}\right] \backslash C$ with zero probability.

Lemma 6: For every $x \in C$, there exists a unique $\gamma(x) \in$ $[0, q]$ such that

$$
f_{x}(\gamma(x))=g(\tilde{p}) .
$$

Also, $\gamma(\tilde{p})=0$ and $\gamma\left(v_{T}\right)=q$.

Proof: First, note that by (3) and Lemma 5:

$$
f_{v_{T}}(q)=h\left(v_{T}\right)=g(\tilde{p}) .
$$

Now, fix an $x \in C$. By (4):

$$
\begin{aligned}
f_{x}(0) & =g(x) \\
& \geq g(\tilde{p})(\text { by }(10), \text { since } x \in C) .
\end{aligned}
$$

Also, by (3):

$$
\begin{aligned}
f_{x}(q)= & h(x) \\
\leq & h\left(v_{T}\right) \quad\left(\text { since } v_{T}\right. \text { is the smallest } \\
& \left.\quad \text { maximizer of } h(.) \text { and } x \leq v_{T}\right) \\
= & g(\tilde{p})(\text { by }(12))
\end{aligned}
$$


By part 1 of Lemma $3, f_{x}(y)$ is continuous in $y$. So by (13), (14) and the intermediate value theorem [3], the equation $f_{x}(y)=g(\tilde{p})$ has a solution $y=\gamma(x) \in[0, q]$. Also, by Lemma 4, this root is unique.

Now, by (4), $f_{\tilde{p}}(0)=g(\tilde{p})$. So $\gamma(\tilde{p})=0$. Also, by (12), $f_{v_{T}}(q)=g(\tilde{p})$. So $\gamma\left(v_{T}\right)=q$.

Now, we state a general analytic fact, which is proved in the Appendix.

Fact 1: Let $F(x, y)$ be any real-valued continuous function, where $x$ and $y$ are real, and $[a, b]$ be an interval such that for every $x \in[a, b]$, there exists a unique $y=\gamma(x)$ such that

$$
F(x, \gamma(x))=\alpha
$$

where $\alpha$ is a constant. Then the function $\gamma($.$) is continuous$ on $[a, b]$.

Now, let $C$ be as in (10). Since $g($.$) is continuous, C$ is closed. So $C$ is the union of a set of disjoint closed intervalslet $C=\cup_{i \in \lambda} C_{i}$, where $\lambda$ is some set of indices and $C_{i}=$ $\left[a_{i}, b_{i}\right]$.

Fix an $i \in \lambda$. By Lemma 6 , for every $x \in C_{i}$, there exists a unique $\gamma(x) \in[0, q]$ such that $f_{x}(\gamma(x))=g(\tilde{p})$. By part 1 of Lemma 3, the function $f_{x}(y)$ is continuous in $x$ and $y$. So by Fact $1, \gamma($.$) is continuous on C_{i}$.

Thus, we have shown the following:

Lemma 7: $\gamma($.$) is continuous on each C_{i}, i \in \lambda$.

By definition of the function $f_{x}(y)$ and by (11), for every $x \in C, \gamma(x)$ is a value such that if $P\left\{p_{j}^{\prime} \leq x\right\}=\gamma(x), j \neq i$, then a price of $p_{i}=x$ fetches primary $i$ a payoff of exactly $g(\tilde{p})$, which is the symmetric NE best response payoff. Since $P\left\{p_{j}^{\prime} \leq x\right\}=\phi(x) \forall j$ in the symmetric NE, this suggests $\gamma($.$) (suitably extended to the region x \notin C$ ) as a candidate for the symmetric NE pseudo-price selection strategy d.f. $\phi($.$) .$ But $\gamma(x)$ itself need not be a valid d.f. since it is not nondecreasing in general as shown in Fig. 1. So a natural idea is to consider the function:

$$
\phi_{N E}(x)= \begin{cases}\max \{\gamma(y): y \in C, y \leq x\}, & x \geq \tilde{p} \\ 0, & x<\tilde{p}\end{cases}
$$

obtained by replacing the portions where $\gamma(x)$ is smaller than

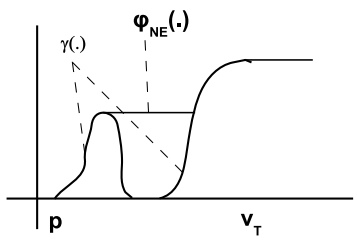

Fig. 1. The figure shows $\phi_{N E}($.$) and \gamma($.$) versus price.$

$\gamma(y)$ for some $y<x$ (and $y \in C$ ) by horizontal segments as illustrated in Fig. 1.

Theorem 1: The strategy profile in which each primary uses the pseudo-price selection strategy $\phi()=.\phi_{N E}($.$) is a NE.$

Proof: By (16), the function $\phi_{N E}($.$) is non-decreasing$ on $\left[\tilde{p}, v_{T}\right]$. Also, by Lemma 7 and (16), it is continuous on $\left[\tilde{p}, v_{T}\right]$. By Lemma $6, \gamma(x) \in[0, q] \forall x \in C$. So by (16):

$$
0 \leq \phi_{N E}(x) \leq q \quad \forall x \in[c, \bar{v}]
$$

Also, since $\gamma(\tilde{p})=0$ and $\gamma\left(v_{T}\right)=q$ (see Lemma 6), and by (16) and (17):

$$
\phi_{N E}(x)= \begin{cases}0, & x \leq \tilde{p} \\ q, & v_{T} \leq x \leq \bar{v}\end{cases}
$$

Thus, $\phi_{N E}($.$) is a valid pseudo-price d.f. and its support set$ is a subset of $\left[\tilde{p}, v_{T}\right]$.

Suppose every primary uses the strategy $\phi_{N E}($.$) to select$ its pseudo-price. By definition of $f_{x}(y)$, if primary $i$ sets a price of $p_{i}=x$, it gets an expected payoff of:

$$
E\left\{u_{i}\left(x, \psi_{-i}\right)\right\}=f_{x}\left(\phi_{N E}(x)\right) .
$$

By (16), $\phi_{N E}(x) \geq \gamma(x)$ for all $x \in\left[\tilde{p}, v_{T}\right]$. Case (i): Suppose $x \in\left[\tilde{p}, v_{T}\right] \backslash C$. Then by (19):

$$
\begin{aligned}
E\left\{u_{i}\left(x, \psi_{-i}\right)\right\} & =f_{x}\left(\phi_{N E}(x)\right) \\
\leq & f_{x}(0) \quad(\text { by Lemma } 4 \text { and (17)) } \\
= & g(x) \quad(\text { by }(4)) \\
< & g(\tilde{p}) \quad\left(\text { since } x \in\left[\tilde{p}, v_{T}\right] \backslash C\right. \\
& \quad \text { and by (10)) }
\end{aligned}
$$

Case (ii): Suppose $x \in C$ and $\phi_{N E}(x)=\gamma(x)$. Then by (19) and (11), $E\left\{u_{i}\left(x, \psi_{-i}\right)\right\}=f_{x}(\gamma(x))=g(\tilde{p})$.

Case (iii): Now, suppose $x \in C$ and $\phi_{N E}(x)>\gamma(x)$. Then by (19), Lemma 4 and (11):

$$
E\left\{u_{i}\left(x, \psi_{-i}\right)\right\}<f_{x}(\gamma(x))=g(\tilde{p})
$$

Also, $x$ is part of an interval of constancy of $\phi_{N E}(x)$; so primaries play prices around $x$ with 0 positive probability.

Case (iv): Suppose $x<\tilde{p}$. Then by (18), $\phi_{N E}(x)=0$. So by (19),

$$
\begin{aligned}
E\left\{u_{i}\left(x, \psi_{-i}\right)\right\} & =f_{x}(0) \\
& =g(x) \quad(\text { by }(4)) \\
& <g(\tilde{p}) \quad(\text { by Lemma } 5)
\end{aligned}
$$

Case (v): Suppose $v_{T} \leq x \leq \bar{v}$. Then by (18), $\phi_{N E}(x)=q$. So by (19),

$$
\begin{aligned}
E\left\{u_{i}\left(x, \psi_{-i}\right)\right\} & =f_{x}(q) \\
& =h(x) \quad(\text { by }(3)) \\
& \leq h\left(v_{T}\right) \quad(\text { by }(6)) \\
& =g(\tilde{p}) \quad(\text { by }(12))
\end{aligned}
$$

Now, since $\phi_{N E}($.$) is non-decreasing and continuous on$ $[c, \bar{v}]$, it has alternating intervals of constancy and strict increase. Also, note that a primary who uses the d.f. $\phi_{N E}($. to select its pseudo-price plays prices in the intervals of constancy with 0 probability and in the intervals of strict increase with positive probability. Now, by (18), the intervals $[c, \tilde{p}]$ and $\left[v_{T}, \bar{v}\right]$ (Cases (iv) and (v) respectively) are intervals of constancy of $\phi_{N E}($.$) . Also, it can be checked using (16)$ that the intervals which lie in the regions $\left[\tilde{p}, v_{T}\right] \backslash C$ and $\left\{x \in C: \phi_{N E}(x)>\gamma(x)\right\}$ (Cases (i) and (iii) respectively) are also regions of constancy. Thus, only intervals that lie in the region $\left\{x \in C: \phi_{N E}(x)=\gamma(x)\right\}$ (Case (ii)) can possibly be intervals of strict increase of $\phi_{N E}($.$) .$ 
By Cases (i) to (v), primary $i$ gets a payoff of at most $g(\tilde{p})$ at any price. Also, as shown in the previous paragraph, it can only play intervals in the region $\left\{x \in C: \phi_{N E}(x)=\gamma(x)\right\}$ (Case (ii)) with positive probability. Its expected payoff is $g(\tilde{p})$, the maximum possible, at a price in this region by Case (ii). Hence $\phi_{N E}($.$) is a best response for primary i$. The result follows.

Note that in the proof of Theorem 1, we have shown the following:

Lemma 8: In the symmetric NE in which every primary uses the strategy $\phi_{N E}($.$) , each primary gets an expected payoff$ of $g(\tilde{p})$.

\section{B. Uniqueness of Symmetric NE}

Now, we show that the NE in Theorem 1 is the unique symmetric NE.

Let the functions $f_{x}(y), h(),. g(),. \gamma($.$) and \phi_{N E}($.$) be as$ in (5), (3), (4), Lemma 6 and (16) respectively. Also, let $v_{T}$, $\tilde{p}$ and the set $C$ be as in (6), Lemma 5 and (10) respectively.

Consider a symmetric NE under which every primary uses the d.f. $\hat{\psi}($.$) to select the price, and let \hat{\phi}_{N E}()=.q \hat{\psi}($.$) be$ the corresponding pseudo-price d.f.

Let $v_{T}^{\prime}$ be the upper endpoint of the support set of $\hat{\psi}($.$) :$

$$
v_{T}^{\prime}=\inf \{x: \hat{\psi}(x)=1\} .
$$

Lemma 9: $v_{T}^{\prime}=v_{T}$. Also, $v_{T}$ is a best response for each primary in the symmetric NE.

Thus, the upper endpoint of the support set of $\hat{\psi}($.$) is v_{T}$.

Proof: As in the proof of Lemma $1, \hat{\psi}($.$) is continuous.$ Also, note that by (26), each primary plays prices in $\left[v_{T}^{\prime}-\right.$ $\left.\epsilon, v_{T}^{\prime}\right]$ with positive probability for every $\epsilon>0$. Hence, $v_{T}^{\prime}$ is a best response for each primary $i$.

To reach a contradiction, suppose $v_{T}^{\prime}>v_{T}$. Then by (26), $\hat{\psi}\left(v_{T}\right)<1$ and hence

$$
\hat{\phi}_{N E}\left(v_{T}\right)<q .
$$

Similar to the derivation of (19):

$$
\begin{aligned}
E\left\{u_{i}\left(v_{T}, \hat{\psi}_{-i}\right)\right\} & =f_{v_{T}}\left(\hat{\phi}_{N E}\left(v_{T}\right)\right) \\
& >f_{v_{T}}(q) \quad \text { by (27) and Lemma 4) } \\
& =h\left(v_{T}\right) \text { (by (3)) } \\
& \geq h\left(v_{T}^{\prime}\right) \quad \text { by (6)) } \\
& =E\left\{u_{i}\left(v_{T}^{\prime}, \hat{\psi}_{-i}\right)\right\}
\end{aligned}
$$

where (29) follows from (28) similar to the derivation of (23). Thus, $E\left\{u_{i}\left(v_{T}, \hat{\psi}_{-i}\right)\right\}>E\left\{u_{i}\left(v_{T}^{\prime}, \hat{\psi}_{-i}\right)\right\}$, which contradicts the fact that $v_{T}^{\prime}$ is a best response. Thus, $v_{T}^{\prime}>v_{T}$ is not possible.

Now suppose $v_{T}^{\prime}<v_{T}$. Then $\hat{\psi}\left(v_{T}\right)=\hat{\psi}\left(v_{T}^{\prime}\right)=1$ by (26); so $\hat{\phi}_{N E}\left(v_{T}\right)=\hat{\phi}_{N E}\left(v_{T}^{\prime}\right)=q$. Similar to the derivation of (23):

$$
\begin{aligned}
E\left\{u_{i}\left(v_{T}, \hat{\psi}_{-i}\right)\right\} & =h\left(v_{T}\right) \\
& >h\left(v_{T}^{\prime}\right) \quad(\text { by }(6)) \\
& =E\left\{u_{i}\left(v_{T}^{\prime}, \hat{\psi}_{-i}\right)\right\}
\end{aligned}
$$

which is again a contradiction. Thus, $v_{T}^{\prime}<v_{T}$ is not possible and hence $v_{T}^{\prime}=v_{T}$.
Now we are ready to prove the uniqueness of the symmetric NE strategy $\phi_{N E}($.$) in Theorem 1$.

Theorem 2: $\phi_{N E}($.$) constitutes the unique symmetric NE$ strategy.

Proof: Consider a symmetric NE in which every primary uses the price (respectively, pseudo-price) selection strategy $\hat{\psi}($.$\left.) (respectively, \hat{\phi}_{N E}().\right)$. We will show that $\hat{\phi}_{N E}()=$. $\phi_{N E}($.$) .$

As in the proof of Lemma 1, $\hat{\psi}($.$) is continuous. Also, by$ Lemma $9, v_{T}$ is the upper endpoint of the support set of $\hat{\psi}($. and is a best response for each primary $i$ in the symmetric NE. By definition of $f_{x}(y)$, the payoff that each primary $i$ gets at price $x$ in the NE is:

$$
E\left\{u_{i}\left(x, \hat{\psi}_{-i}\right)\right\}=f_{x}\left(\hat{\phi}_{N E}(x)\right)
$$

Also, similar to the derivation of (23), the payoff that each primary $i$ gets at price $v_{T}$ is:

$$
E\left\{u_{i}\left(v_{T}, \hat{\psi}_{-i}\right)\right\}=h\left(v_{T}\right)=g(\tilde{p}),
$$

where the second equality follows from (12). Since $v_{T}$ is a best response, each primary gets an expected payoff of $g(\tilde{p})$ in the NE.

Now, for a price $x<\tilde{p}$, by (30), primary $i$ gets a payoff of:

$$
\begin{aligned}
E\left\{u_{i}\left(x, \hat{\psi}_{-i}\right)\right\} & =f_{x}\left(\hat{\phi}_{N E}(x)\right) \\
& \leq f_{x}(0) \quad(\text { by Lemma } 4) \\
& <g(\tilde{p})
\end{aligned}
$$

where (33) follows from (32) similar to the derivation of (22). Thus, primaries do not play prices below $\tilde{p}$ in the NE and hence $\hat{\phi}_{N E}(\tilde{p})=0$.

Similar to the derivation of (20), it can be shown that for $x \in\left[\tilde{p}, v_{T}\right] \backslash C, E\left\{u_{i}\left(x, \hat{\psi}_{-i}\right)\right\}<g(\tilde{p})$ and hence $x$ is not a best response. Thus, only prices in $C$ can possibly be best responses.

If $x_{0}$ is a best response for primary $i$, then by (30):

$$
E\left\{u_{i}\left(x_{0}, \hat{\psi}_{-i}\right)\right\}=f_{x_{0}}\left(\hat{\phi}_{N E}\left(x_{0}\right)\right)=g(\tilde{p}) .
$$

By (34) and Lemma 6:

$$
\hat{\phi}_{N E}\left(x_{0}\right)=\gamma\left(x_{0}\right),
$$

Now, since $\hat{\phi}_{N E}($.$) is continuous on [c, \bar{v}]$, it consists of alternating intervals of strict increase and constancy. If $\left[a_{s}, b_{s}\right]$ is an interval of strict increase, then each $x \in\left[a_{s}, b_{s}\right]$ is a best response; so $\hat{\phi}_{N E}(x)=\gamma(x)$ by (35). Thus,

$\hat{\phi}_{N E}(x) \leq \max \{\gamma(y): y \in C, y \leq x\}=\phi_{N E}(x), \forall x \in\left[a_{s}, b_{s}\right]$.

where the equality follows by (16).

Now, let $\left[a_{c}, b_{c}\right]$ be a maximal interval of constancy of $\hat{\phi}_{N E}($.$) such that \hat{\phi}_{N E}\left(a_{c}\right)>0$. Note that $a_{c}$ is the right endpoint of an interval of strict increase ${ }^{9}$. So by continuity of $\hat{\phi}_{N E}(),. a_{c}$ is a best response and hence $\hat{\phi}_{N E}\left(a_{c}\right)=\gamma\left(a_{c}\right)$

\footnotetext{
${ }^{9}$ Note that $\hat{\phi}_{N E}\left(a_{c}\right)>0$ implies that there exists an interval of strict increase of $\hat{\phi}_{N E}($.$) to the left of a_{c}$.
} 
by (35). So for all $x \in\left[a_{c}, b_{c}\right], \hat{\phi}_{N E}(x)=\hat{\phi}_{N E}\left(a_{c}\right)=\gamma\left(a_{c}\right)$ Thus,

$\hat{\phi}_{N E}(x) \leq \max \{\gamma(y): y \in C, y \leq x\}=\phi_{N E}(x), \forall x \in\left[a_{c}, b_{c}\right]$

where the equality follows by (16).

By (36) and (37):

$$
\hat{\phi}_{N E}(x) \leq \phi_{N E}(x) \forall x \in[c, \bar{v}] .
$$

It remains to show that $\hat{\phi}_{N E}(x) \geq \phi_{N E}(x)$ for all $x \in$ $[c, \bar{v}]$. To reach a contradiction, suppose $\hat{\phi}_{N E}(x)<\phi_{N E}(x)$ for some $x \in[c, \bar{v}]$. Let:

$$
x_{l}=\inf \left\{x: \hat{\phi}_{N E}(x)<\phi_{N E}(x)\right\} .
$$

Then for all $x<x_{l}, \hat{\phi}_{N E}(x)=\phi_{N E}(x)$. So by continuity of $\hat{\phi}_{N E}($.$) and \phi_{N E}($.$) ,$

$$
\hat{\phi}_{N E}\left(x_{l}\right)=\phi_{N E}\left(x_{l}\right) .
$$

Also, by (39), there exists an $x_{0}=x_{l}+\epsilon$, for some small $\epsilon>0$, such that:

$$
\hat{\phi}_{N E}\left(x_{0}\right)<\phi_{N E}\left(x_{0}\right) .
$$

and $\left[x_{l}, x_{0}\right]$ is an interval of strict increase of $\phi_{N E}($.$) . In$ particular, $x_{0}$ is a best response of primary $i$ when the other primaries use $\phi_{N E}($.$) .$

Now, by (30), the expected payoff of primary $i$ for price $p_{i}=x_{0}$ when other primaries play $\hat{\phi}_{N E}($.$) is:$

$$
\begin{aligned}
f_{x_{0}}\left(\hat{\phi}_{N E}\left(x_{0}\right)\right) & >f_{x_{0}}\left(\phi_{N E}\left(x_{0}\right)\right) \quad \text { (by (41) and Lemma 4) } \\
& =g(\tilde{p})
\end{aligned}
$$

where (42) follows from the fact that $x_{0}$ is a best response of primary $i$ when the other primaries use $\phi_{N E}($.$) and Lemma 8$. This contradicts the fact that the maximum payoff that primary $i$ can get when the other primaries use $\hat{\phi}_{N E}($.$) is g(\tilde{p})$. Thus,

$$
\hat{\phi}_{N E}(x) \geq \phi_{N E}(x) \forall x \in[c, \bar{v}]
$$

By (38) and (43), $\hat{\phi}_{N E}(x)=\phi_{N E}(x) \forall x \in[c, \bar{v}]$ and the result follows.

\section{RANDOM Number of SeCONDARIES}

In Section III, we explicitly computed the symmetric NE and showed its uniqueness for the case of one secondary. We now generalize our results to the model described in Section II-A, in which there are a random number, $K$, of secondaries.

\section{A. Primary Secondary Matching Scheme}

Since there may be multiple secondaries with possibly different valuations, after the primaries reveal the prices they are willing to sell at, the number of secondaries is known, and the secondaries reveal their valuations, there are in general different possible schemes for matching primaries with the secondaries who buy bandwidth from them. Consider a scenario in which there are $k$ secondaries, where $k$ is a constant. Let $p_{(1)}^{\prime} \leq p_{(2)}^{\prime} \leq \ldots \leq p_{(n)}^{\prime}$ be the pseudoprices $p_{1}^{\prime}, \ldots, p_{n}^{\prime}$ of the primaries in increasing order. Also, let $v^{(1)} \geq v^{(2)} \geq \ldots \geq v^{(k)}$ be the valuations of the secondaries in decreasing order.

Let $\mathcal{A}$ be the set of all possible schemes of matching primaries with secondaries such that bandwidth is never bought from a primary if the bandwidth of a different primary who offers a lower pseudo-price remains unsold. Note that under every scheme in $\mathcal{A}$, the bandwidth of the primaries with the smallest $i$ pseudo-prices $p_{(1)}^{\prime}, \ldots, p_{(i)}^{\prime}$ is sold, for some $i \in\{0,1, \ldots, n\}$, and the bandwidth of the rest of the primaries is not sold. Let $W$ be the scheme in which the secondary with the highest valuation $v^{(1)}$ buys from the primary with the lowest price $p_{(1)}^{\prime}$ (if $p_{(1)}^{\prime} \leq v^{(1)}$ ), the secondary with the second-highest valuation $v^{(2)}$ buys from the primary with the second-lowest price $p_{(2)}^{\prime}$ (if $p_{(2)}^{\prime} \leq v^{(2)}$ ) and so on. Ties are broken at random. Note that $W \in \mathcal{A}$.

For example, suppose $n=4, k=3$, the pseudo-prices of the primaries in increasing order are $p_{(1)}^{\prime}=1, p_{(2)}^{\prime}=2, p_{(3)}^{\prime}=$ $3, p_{(4)}^{\prime}=4$ and the valuations of the secondaries in decreasing order are $v^{(1)}=3.5, v^{(2)}=2.5, v^{(3)}=1.5$. For the scheme $W$, the following table shows the valuation of the secondary who buys bandwidth from each primary (a "." indicates that the corresponding primary's bandwidth is unsold):

\begin{tabular}{|c|c|}
\hline Primary's price & Secondary's valuation \\
\hline 1 & 3.5 \\
2 & 2.5 \\
3 & - \\
4 & - \\
\hline
\end{tabular}

Consider another scheme in $\mathcal{A}$ under which the following matching of primaries and secondaries is done for the above example:

\begin{tabular}{|c|c|}
\hline Primary's price & Secondary's valuation \\
\hline 1 & 1.5 \\
2 & 2.5 \\
3 & 3.5 \\
4 & - \\
\hline
\end{tabular}

The above tables show that the second scheme is more "efficient" than the scheme $W$ in the sense that more primaries sell their bandwidth. In fact, the following lemma shows that in this sense the scheme $W$ is the worst-case or least efficient scheme in $\mathcal{A}$.

Lemma 10: For any given set of pseudo-prices of the primaries and valuations of the secondaries, out of all the schemes in $\mathcal{A}$, the bandwidth of the fewest number of primaries is sold under the scheme $W$.

Proof: Fix $p_{(1)}^{\prime}, \ldots, p_{(n)}^{\prime}$ and $v^{(1)}, \ldots, v^{(k)}$. Suppose, under the scheme $W$, the bandwidth of the primaries with psedo-prices $p_{(1)}^{\prime}, \ldots, p_{(i)}^{\prime}$ is sold. By definition of $W$, these $i$ primaries sell their bandwidth to the secondaries with the $i$ largest valuations $v^{(1)}, \ldots, v^{(i)}$ and the primary with pseudoprice $p_{(i)}^{\prime}$ sells to the secondary with the smallest valuation $v^{(i)}$ out of these. Thus, $v^{(i)} \geq p_{(i)}^{\prime}$ and hence:

$$
v^{(j)} \geq p_{(i)}^{\prime}, j=1, \ldots, i .
$$

Now, consider an arbitrary scheme $A \in \mathcal{A}$, and suppose, to reach a contradiction, that under $A$, only the bandwidth of the primaries with pseudo-prices $p_{(1)}^{\prime}, \ldots, p_{\left(i^{\prime}\right)}^{\prime}$ is sold for some $i^{\prime}<i$. Hence, at most $i^{\prime}$ out of the secondaries with valuations 
$v^{(1)}, \ldots, v^{(i)}$ buy bandwidth under $A$ and hence at least one of these secondaries does not buy bandwidth. However, by (44), the valuation of such a secondary is $\geq$ the pseudo-price $p_{\left(i^{\prime}+1\right)}$, which contradicts the fact that the bandwidth of the primary with pseudo-price $p_{\left(i^{\prime}+1\right)}$ remains unsold.

The following lemma is an immediate consequence of Lemma 10.

Lemma 11: Out of all the schemes in $\mathcal{A}$ and for any given set of pseudo-price distributions of the primaries and distributions of the valuations of the secondaries, given that a primary $i$ has unused bandwidth and sets price $p_{i}=x$, the probability that its bandwidth is sold, and hence its expected payoff, is minimized for the scheme $W$.

We assume that primaries do not know the scheme that will be used to match the primaries and secondaries, and hence, each primary, so as to maximize its worst-case payoff, selects its price distribution assuming that the scheme $W$ will be used. Note that the scheme $W$ has also been used to match the buyers and sellers in a marketplace in [14].

\section{B. Analysis}

We now generalize the analysis in Section III to a random number, $K$, of secondaries. First, it is easy to see that Lemma 1 readily generalizes to the case of a random number of secondaries. Now, recall from Section II-A that the valuations of the secondaries are i.i.d., and each has the d.f. $G($.$) . When$ there are $K=k$ secondaries, for $i=1, \ldots, k$, let $v_{k}^{(i)}$ be the $i$ 'th largest valuation of a secondary and let $G_{k}^{(i)}($.$) be the$ d.f. of $v_{k}^{(i)}$. Recall that algebraic expressions for the functions $G_{k}^{(i)}(),. i=1, \ldots, k$ can be easily obtained in terms of the function $G($.$) [7]. The following lemma provides some simple$ properties of the functions $G_{k}^{(i)}(),. i=1, \ldots, k$.

Lemma 12: $G_{k}^{(i)}(),. i=1, \ldots, k$ are continuous. Also:

$$
\begin{gathered}
G_{k}^{(i)}(x)=0, x \leq \underline{v} ; i=1, \ldots, k \\
G_{k}^{(i)}(x)=1, x \geq \bar{v} ; i=1, \ldots, k \\
G_{k}^{(1)}(x) \leq G_{k}^{(2)}(x) \leq \ldots \leq G_{k}^{(k)}(x), x \in[c, \bar{v}] .
\end{gathered}
$$

Proof: The continuity of $G_{k}^{(i)}(),. i=1, \ldots, k$ follows from the continuity of $G($.$) . Also, (45) and (46) follow from$ the fact that $P\left(\underline{v} \leq v_{j} \leq \bar{v}\right)=1$ for every buyer $j$. Finally, we get (47) from the fact that $v_{k}^{(1)} \geq v_{k}^{(2)} \geq \ldots \geq v_{k}^{(k)}$.

Let $f_{x}(y)$ be as defined just before (2) in Section III and $h($.$) and g($.$) be as in (3) and (4) respectively. In Lemma 2,$ we derived an expression for $f_{x}(y)$ for the case of one secondary. The following lemma generalizes that expression to $K$ secondaries.

Lemma 13:

$$
f_{x}(y)=\sum_{k=0}^{k_{\max }} \alpha_{k} f_{x, k}(y)
$$

where ${ }^{10}$,

$$
f_{x, k}(y)=(x-c) \sum_{i=1}^{k}\left(1-G_{k}^{(i)}(x)\right)\left(\begin{array}{c}
n-1 \\
i-1
\end{array}\right) y^{i-1}(1-y)^{n-i}
$$

Proof: Let $f_{x, k}(y)$ be the value of $f_{x}(y)$ conditioned on the event $K=k$; then clearly $f_{x}(y)$ is given by (48). We now show (49). Let $K=k$. Let $Z$ be the number of primaries out of primaries $\{1, \ldots, n\} \backslash m$ for which the pseudo-price $p_{j}^{\prime} \leq x$. By (2), the events $\left\{p_{j}^{\prime} \leq x\right\}, j \in\{1, \ldots, n\} \backslash m$ are independent Bernoulli events with success probability $y$ each. So:

$$
P(Z=i-1)=\left(\begin{array}{c}
n-1 \\
i-1
\end{array}\right) y^{i-1}(1-y)^{n-i} .
$$

Also, under the scheme $W$, if $Z=i-1$ for some $i \in$ $\{1, \ldots, k\}$, then primary $m$ 's bandwidth is sold iff ${ }^{11} i$ or more secondaries have valuations $\geq x$; the probability of the latter event is:

$$
1-G_{k}^{(i)}(x)
$$

If $Z \geq k$, then primary $m$ 's bandwidth is not sold. Conditioning on $Z$ and using (50) and (51), we get that the probability that primary $m$ 's bandwidth is sold given that it sets a price $p_{m}=x$ equals the summation in (49). This, combined with the fact that if primary $m$ 's bandwidth is sold at price $p_{m}=x$, then it gets a payoff of $x-c$, gives (49).

Let $v_{T}$ be defined as in (6). The following lemma, proved in the Appendix, generalizes the properties of $f_{x}(y)$ that were shown for the case of one secondary.

Lemma 14: The properties of $f_{x}(y)$ in Lemma 3 and Lemma 4 hold for the case of $K$ secondaries.

Now, the analysis in Section III after Lemma 4 does not use the expression for $f_{x}(y)$ and relies only on the properties of $f_{x}(y)$ in Lemmas 3 and 4. Since these properties go through for the case of $K$ secondaries by Lemma 14, the analysis in Section III after Lemma 4 generalizes to the case of $K$ secondaries. In particular, we define $\tilde{p}, C$, the function $\gamma($. and the function $\phi_{N E}($.$) as in Lemma 5, (10), Lemma 6$ and (16) respectively. Theorems 1 and 2 generalize to the case of $K$ secondaries and provide the unique symmetric NE strategy.

\section{Discussion on Structure of Symmetric NE STRATEGY}

As noted after Lemma 1, there does not exist a pure-strategy symmetric NE in the game in this paper. This is similar to the case in which the valuations of all the secondaries are constant and equal (see Section II-D), where there is a unique symmetric NE that is of mixed-strategy type.

As explained in Section II-D, when the valuations of all the secondaries are constant and equal, the symmetric NE price selection strategy is contiguous. However, we now show by providing an example that when the valuations of the secondaries are random, the symmetric NE price selection

\footnotetext{
${ }^{10}$ Recall that for integers $a, b \geq 0,\left(\begin{array}{c}a \\ b\end{array}\right)$ equals 0 if $b>a$.

${ }^{11}$ Note that by Lemma 1 , the probability that a primary sets a price of exactly $y$ is 0 .
} 
strategy can be non-contiguous, even when there is only one secondary, i.e., $K=1$.

Let $n=2, c=0, \underline{v}=1, \bar{v}=3, q=\frac{1}{8}$ and

$$
G(x)= \begin{cases}\frac{1}{2}(x-1), & 1 \leq x \leq 2 \\ \frac{1}{2}, & 2<x \leq \frac{5}{2} \\ x-2, & \frac{5}{2}<x \leq 3\end{cases}
$$

By (4), (5) and using $c=0$, we get $g(x)=x(1-G(x))$.

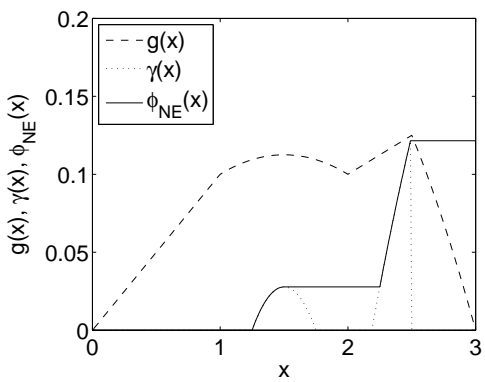

Fig. 2. The figure plots the functions $g(),. \gamma($.$) and \phi_{N E}($.$) versus the price$ $x$ for the example in Section V. The function $g($.$) is scaled down by a factor$ of 10 for clarity.

The function $g($.$) is plotted in Fig. 2. Also, it can be checked$ that $\tilde{p}=\frac{5}{4}$ and $v_{T}=\frac{5}{2}$. Fig. 2 also plots the function $\gamma($.$) and the symmetric NE pseudo-price selection strategy$ $\phi_{N E}($.$) and shows that the latter has an interval of constancy$ $([1.5,2.25])$ within $\left[\tilde{p}, v_{T}\right]$; hence, the symmetric NE price selection strategy is not contiguous. The reason the interval of constancy arises is as follows. Fig. 2 shows that within the interval $[1.5,2.25]$, there is a sub-interval $([1.75,2.15])$ in which $g(x)<g(\tilde{p})$. So with $C$ as in (10), this subinterval is not in $C$. Hence, each primary plays prices in this sub-interval with zero probability (see Case (i) in the proof of Theorem 1), and hence $\phi_{N E}($.$) is constant over the$ interval $[1.75,2.15]$. The rest of the interval $[1.5,2.25]$ (i.e., $(1.5,1.75) \cup(2.15,2.25))$ is in $C$; however, $\gamma(x)<\phi_{N E}(x)$ for $x$ in this region and hence $\phi_{N E}($.$) is constant over this$ region as well (see Case (iii) in the proof of Theorem 1).

\section{NUMERICAL STUdiES}

In this section, we study via numerical computations, the dependence of the structure of the symmetric NE strategy and the mean price of bandwidth charged by each primary under the symmetric NE on different parameters. We consider a scenario in which the valuation of each secondary is uniformly distributed in the range $[\underline{v}, \bar{v}]$. Throughout, we use the parameter values $c=0, \underline{v}=1, \bar{v}=6$ and $n=10$.

In the symmetric $\mathrm{NE}$, each primary uses the d.f. $\phi_{N E}($. studied in Section IV-B to select its pseudo-price. The top plot in Fig. 3 shows the function $\phi_{N E}($.$) for the case when the$ number of secondaries is a constant, $K=8$. The bottom plot in Fig. 3 shows $\phi_{N E}($.$) when K$ takes values in $\{0,1, \ldots, 16\}$ uniformly at random. Note that the mean number of secondaries $E(K)=8$ for both plots, and the other parameters are also the same. The plots show that primaries randomize over a larger range of prices when the variance in the number of secondaries is larger. Intuitively this is because, primaries tend to prefer low prices when the number of secondaries is small since they may not find buyers at high prices and vice versa. So when the variance in the number of secondaries is larger, primaries set very low prices as well as very high prices since they are suitable in the event that the number of secondaries is small or high respectively.
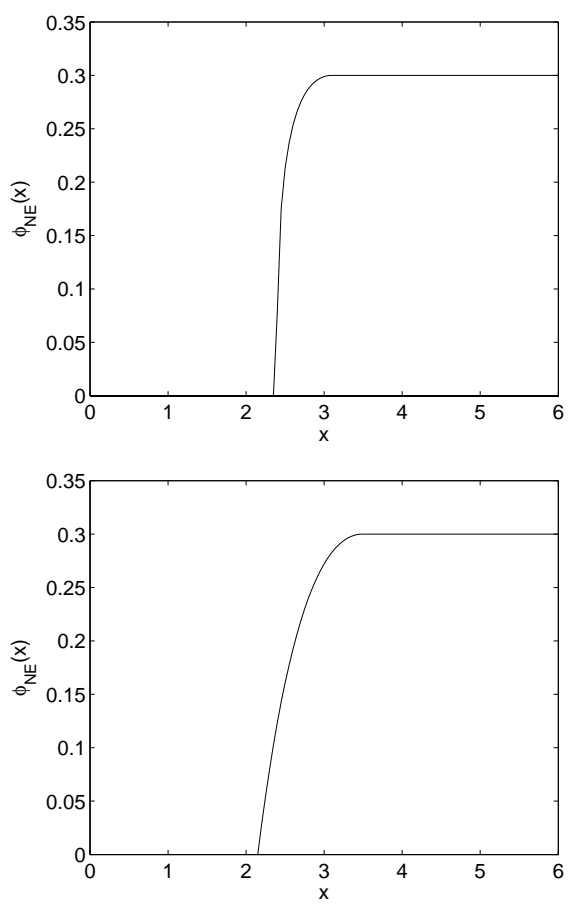

Fig. 3. The top plot shows $\phi_{N E}($.$) versus the price x$ for the case $K=8$ and $q=0.3$. Here, $\tilde{p}=2.4$ and $v_{T}=3.1$. The bottom plot shows $\phi_{N E}($. versus the price $x$ for the case in which $K$ takes values in $\{0,1, \ldots, 16\}$ uniformly at random and $q=0.3$. Here, $\tilde{p}=2.2$ and $v_{T}=3.5$.

For the case in which the number of secondaries is a constant, $K=k$, Fig. 4 plots the mean price of bandwidth charged under the symmetric NE by a primary who has unused bandwidth versus $k$. The figure shows that the mean price is increasing in $k$. This is because, as $k$ increases, the demand for bandwidth increases and hence the primaries are able to find buyers for their bandwidth even when they set high prices. The top plot and bottom plot in Fig. 5 show the mean price versus $q$ for a constant and uniformly distributed number of secondaries respectively. In both plots, the mean price is decreasing in $q$. This is because, as $q$ increases, the expected supply of bandwidth in the market increases and the price competition becomes more intense driving down the prices.

\section{CONCLUSIONS}

We studied price competition among primary users in a CRN, taking into account uncertainty in the availability of bandwidth with the primaries, randomness in the number of secondaries and randomness in the valuations of secondaries for unit bandwidth. We explicitly computed the symmetric $\mathrm{NE}$ and showed its uniqueness. The analysis yields several insights- for example, it shows that randomness in the valuations of secondaries may introduce non-contiguity in the price distributions used by the primaries. We also presented 


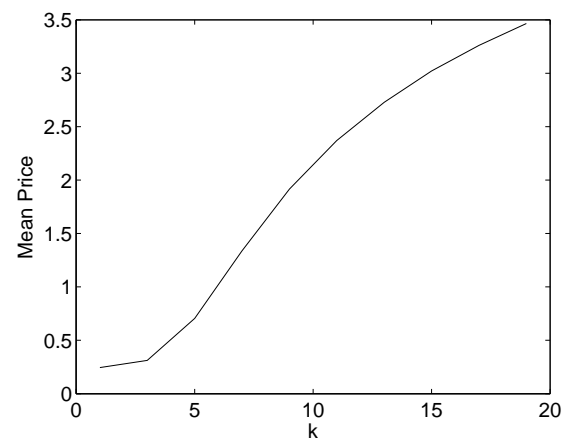

Fig. 4. The figure plots the mean price of bandwidth charged by a primary who has unused bandwidth versus $k$. The parameter value $q=0.5$ is used.
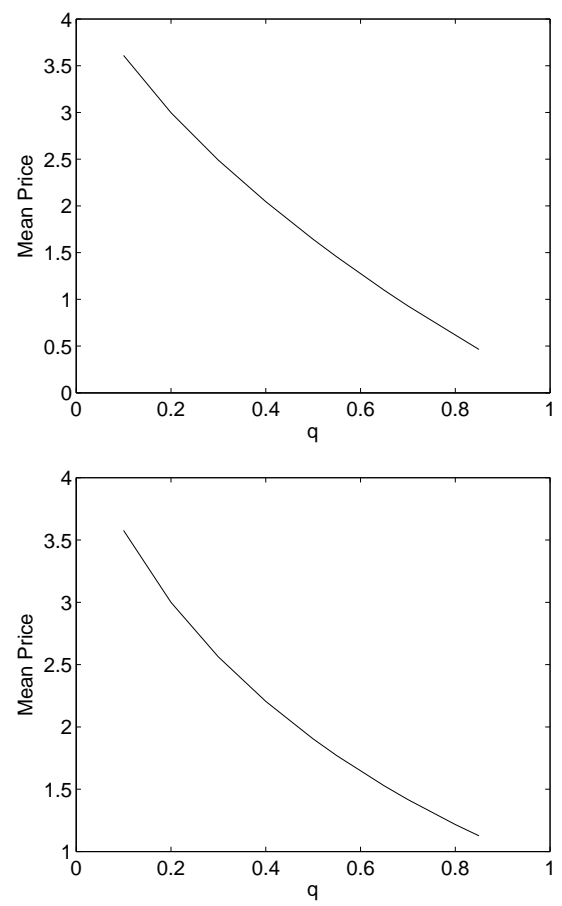

Fig. 5. Both plots show the mean price of bandwidth charged by a primary who has unused bandwidth versus $q$. The number of secondaries is constant, $K=8$, for the top plot and takes values in $\{0,1, \ldots, 16\}$ uniformly at random for the bottom plot.

numerical studies, which provide further insights into the dependence of the symmetric NE strategy and the mean price of bandwidth on different system parameters.

\section{REFERENCES}

[1] I. Akyildiz, W.-Y. Lee, M. Vuran, S. Mohanty "NeXt generation/dynamic spectrum access/cognitive radio wireless networks: a survey". In Comp. Networks, Vol. 50, 13, pp. 2127-59, 2006.

[2] J. E. Walsh, "Existence of Every Possible Distribution for any Sample Order Statistic", In Statistical Papers, Vol. 10, No. 3, Springer Berlin, Sept. 1969.

[3] W. Rudin, "Principles of Mathematical Analysis", Mc-Graw Hill, Third Edition, 1976.

[4] S.-F. Cheng, D.M. Reeves, Y. Vorobeychik, M.P. Wellman, "Notes on Equilibria in Symmetric Games", In AAMAS-04 Workshop on GameTheoretic and Decision-Theoretic Agents, 2004.

[5] M. Osborne, C. Pitchik, "Price Competition in a Capacity-Constrained Duopoly", J. Econ. Theory, 38(2), 238-260, 1986.
[6] D.M. Kreps, J.A. Scheinkman, "Quantity Precommitment and Bertrand Competition Yield Cournot Outcomes", In Bell Journal of Economics, 14, pp. 326-337, Autumn 1983.

[7] H.A. David, H.N. Nagaraja, "Order Statistics", Wiley, New Jersey, Third Edition, 2003.

[8] M. Janssen, E. Rasmusen "Bertrand Competition Under Uncertainty", In J. Ind. Econ., 50(1): pp. 11-21, March 2002.

[9] S. Kimmel "Bertrand Competition Without Completely Certain Production", Economic Analysis Group Discussion Paper, Antitrust Division, U.S. Department of Justice, 2002.

[10] C. Courcoubetis and R. Weber, "Pricing Communication Networks", John Wiley \& Sons, Ltd. 2003.

[11] O. Ileri, D. Samardzija, T. Sizer, N. B. Mandayam, "Demand Responsive Pricing and Competitive Spectrum Allocation via a Spectrum Policy Server", In Proc. of IEEE DySpan, 2005.

[12] P. Maille, B. Tuffin "Analysis of Price Competition in a Slotted Resource Allocation Game", In Proc. of Infocom, 2008.

[13] P. Maille, B. Tuffin, "Price War with Partial Spectrum Sharing for Competitive Wireless Service Providers", In Proc. of IEEE Globecom, Dec. 2009.

[14] B. Shi, E.H. Gerding, P. Vytelingum, N.R. Jennings, "An Equilibrium Analysis of Competing Double Auction Marketplaces Using Fictitious Play", In Proc. of ECAI, 2010.

[15] Y. Xing, R. Chandramouli, C. Cordeiro, "Price Dynamics in Competitive Agile Spectrum Access Markets", In IEEE JSAC, Vol. 25, No. 3, April 2007.

[16] D. Niyato, E. Hossain, "Competitive Pricing for Spectrum Sharing in Cognitive Radio Networks: Dynamic Game, Inefficiency of Nash Equilibrium, and Collusion", IEEE JSAC, Vol. 26, No. 1, 2008.

[17] D. Niyato, E. Hossain, Z. Han, "Dynamics of Multiple-Seller and Multiple-Buyer Spectrum Trading in Cognitive Radio Networks: A Game-Theoretic Modeling Approach", IEEE TMC, Vol. 8, No. 8, pp. 1009-1022, Aug. 2009.

[18] R. Myerson, "Game Theory: Analysis of Conflict", Harvard University Press, 1997.

[19] A. Mas-Colell, M. Whinston, J. Green, "Microeconomic Theory", Oxford University Press, 1995.

[20] B.S. Everitt, The Cambridge Dictionary of Statistics, 3rd ed., Cambridge University Press, 2006.

[21] G.S. Kasbekar, S. Sarkar, "Spectrum Pricing Games with Bandwidth Uncertainty and Spatial Reuse in Cognitive Radio Networks", in Proc. of MobiHoc, Chicago, IL, USA, Sept. 20-24, 2010, (Technical report: http://www.seas.upenn.edu/ swati/publication.htm)

[22] G.S. Kasbekar, S. Sarkar, "Spectrum Pricing Games with Arbitrary Bandwidth Availability Probabilities", In Proc. of ISIT, St. Petersburg, Russia, July 31-August 5, 2011.

[23] G.S. Kasbekar, S. Sarkar, "Spectrum Pricing Games with Spatial Reuse in Cognitive Radio Networks", Accepted in IEEE JSAC-Special Issue on Game Theory in Wireless Communications.

\section{APPENDIX}

Proofs of Lemmas 3 and 4: Note that the expression for $f_{x}(y)$ in (5) is a special case with $K=1$ of the expression for $f_{x}(y)$ in (48). Below, we directly prove Lemma 14 , from which the proofs of Lemmas 3 and 4 follow.

Proof of Lemma 14: We will show that Lemmas 3 and 4 hold when $f_{x}(y)$ is replaced with $f_{x, k}(y)$ for all $x$ and $y$ throughout in the statement of the lemmas, where $k \geq 1$ is a constant. Then, from (48) and the fact that $\alpha_{0}<1$, it will follow that Lemmas 3 and 4 hold when there are $K$ secondaries, which will prove Lemma 14.

So let $K=k$, where $k \geq 1$ is a constant. By Lemma 12, $G_{k}^{(i)}(),. i=1, \ldots, k$ are continuous. So by (49), it follows that $f_{x, k}(y)$ is continuous in $x$ and $y$, which proves part 1 of Lemma 3 (with the above replacements made). By (45) and (49), for $x \leq \underline{v}$ :

$$
f_{x, k}(y)=(x-c) \sum_{i=1}^{k}\left(\begin{array}{c}
n-1 \\
i-1
\end{array}\right) y^{i-1}(1-y)^{n-i}
$$


from which part 2 of Lemma 3 follows. By (52) and the facts that $\underline{v}>c$ and $0<q<1$, it follows that $h(\underline{v})=f_{\underline{v}, k}(q)>0$. Also, by (46) and (49), $f_{x, k}(y)=0$ for $x \geq \bar{v}$ and hence $h(x)=f_{x, k}(q)=0$ for $x \geq \bar{v}$. This proves part 3 of Lemma 3 .

It remains to prove Lemma 4 (with the above replacements made). By (6) and part 3 of Lemma 3, $h\left(v_{T}\right) \geq h(\underline{v})>0$. Also, by (49) and since $h\left(v_{T}\right)=f_{v_{T}, k}(q)$ by (3), we get $1-G_{k}^{(i)}\left(v_{T}\right)>0$ for at least one value of $i$ in $\{1, \ldots, k\}$. Since $1-G_{k}^{(i)}\left(v_{T}\right)=P\left(v_{k}^{(i)}>v_{T}\right)$, it follows that $P\left(v_{j}>v_{T}\right)>0$, $j=1, \ldots, k$. So $1-G_{k}^{(i)}\left(v_{T}\right)>0$ for all $i=1, \ldots, k$. But for each $i, 1-G_{k}^{(i)}(x)$ is a decreasing function of $x$. Hence:

$$
1-G_{k}^{(i)}(x)>0, x \leq v_{T}, i=1, \ldots, k .
$$

Now, fix an arbitrary $x \in\left[c, v_{T}\right]$. Let $a_{i}=1-G_{k}^{(i)}(x), i=$ $1, \ldots, k$. By (47) and (53):

$$
a_{1} \geq a_{2} \geq \ldots \geq a_{k}>0
$$

Let $b_{i}(y)=\left(\begin{array}{c}n-1 \\ i-1\end{array}\right) y^{i-1}(1-y)^{n-i}$. We have the following property from [2]:

Property 1: For every $1 \leq j \leq n-1, \sum_{i=1}^{j} b_{i}(y)$ is a strictly decreasing function of $y$.

Now, note that for $i \in\{1, \ldots, k\}$ :

$$
a_{i}=\sum_{j=i}^{k-1}\left(a_{j}-a_{j+1}\right)+a_{k} .
$$

Now, by (49), $f_{x, k}(y)=(x-c) T$, where

$$
\begin{aligned}
T & =\sum_{i=1}^{k} a_{i} b_{i}(y) \\
& =\sum_{i=1}^{k}\left\{\sum_{j=i}^{k-1}\left(a_{j}-a_{j+1}\right)+a_{k}\right\} b_{i}(y) \quad(\text { by }(55)) \\
& =a_{k} \sum_{i=1}^{k} b_{i}(y)+\sum_{i=1}^{k} \sum_{j=1}^{k-1}\left(a_{j}-a_{j+1}\right) b_{i}(y) I\{j \geq i\} \\
& =a_{k} \sum_{i=1}^{k} b_{i}(y)+\sum_{j=1}^{k-1}\left(a_{j}-a_{j+1}\right) \sum_{i=1}^{k} b_{i}(y) I\{j \geq i\} \\
& =a_{k} \sum_{i=1}^{k} b_{i}(y)+\sum_{j=1}^{k-1}\left(a_{j}-a_{j+1}\right)\left(\sum_{i=1}^{j} b_{i}(y)\right)
\end{aligned}
$$

By (54), each of the terms $a_{j}-a_{j+1}, j=1, \ldots, k-1$ are nonnegative and $a_{k}>0$; so by Property 1 , the expression in (56) is strictly decreasing in $y$. So $T$, and hence $f_{x, k}(y)=$ $(x-c) T$, is strictly decreasing in $y$ for fixed $x$. The result follows.

Proof of Fact 1: Let $\left\{x_{n}: n=1,2,3, \ldots\right\}$ be any sequence such that $x_{n} \rightarrow x \in[a, b]$. It is sufficient to show that

$$
\lim _{n \rightarrow \infty} \gamma\left(x_{n}\right)=\gamma(x)
$$

To show (57), consider the sequence

$$
y_{n}=\gamma\left(x_{n}\right), n=1,2,3, \ldots
$$

Let $L=\liminf _{n \rightarrow \infty} y_{n}$. Then there exists a subsequence of the sequence $\left\{y_{n}\right\}$, say $\left\{y_{n_{k}}, k=1,2,3, \ldots\right\}$, such that $y_{n_{k}} \rightarrow L$ as $k \rightarrow \infty$. By (15) and (58):

$$
F\left(x_{n_{k}}, y_{n_{k}}\right)=\alpha, k=1,2,3 \ldots
$$

So:

$$
\lim _{k \rightarrow \infty} F\left(x_{n_{k}}, y_{n_{k}}\right)=\alpha .
$$

By continuity of $F($.$) , and using x_{n_{k}} \rightarrow x$ and $y_{n_{k}} \rightarrow L$ :

$$
F(x, L)=\alpha \text {. }
$$

But since $y=\gamma(x)$ is the unique value that satisfies $F(x, y)=$ $\alpha$, we get

$$
L=\gamma(x)
$$

Now, let $U=\limsup _{n \rightarrow \infty} y_{n}$. Similar to the proof of (60), we get:

$$
U=\gamma(x)
$$

By (58), (60) and (61), $\liminf _{n \rightarrow \infty} \gamma\left(x_{n}\right)=\gamma(x)$ and $\lim \sup _{n \rightarrow \infty} \gamma\left(x_{n}\right)=\gamma(x)$, from which (57) follows. This completes the proof. 